ASM Sc. J., 13, 2020

\title{
Orderly Set Graph for Some Metacyclic p- Groups of Odd Prime
}

\author{
Siti Norziahidayu Amzee Zamri ${ }^{1^{*}}$ and Nor Haniza Sarmin ${ }^{2}$ \\ ${ }^{1}$ UniSZA Science and Medicine Foundation Centre, Universiti Sultan Zainal Abidin, \\ 21300 Kuala Nerus, Terengganu, Malaysia \\ ${ }^{2}$ Department of Mathematical Sciences, Faculty of Science, Universiti \\ Teknologi Malaysia, 81310 UTM Johor Bahru, Johor
}

The orderly set graph, denoted by $\Gamma^{O S}$, is a graph where the vertex set is the ordered pair of elements $\left(x_{i}, y_{j}\right) \in \Omega$ where $\{(x, y) \in G \times G: \operatorname{lcm}(|x|,|y|)=p, x y=y x, x \neq y\}$. The ordered pairs in the set $\Omega$ are joined by an edge if and only if the pair consists of elements with similar order. In this research, the metacyclic $p$-groups of odd prime $p$, where $p$ is equal to 3 and 5 are considered. If $G$ acts on $\Omega$ by conjugation action, the orderly set graph of metacyclic $p$-groups where $p$ is an odd prime are found to be a union of complete and empty graph with $n$ number of vertices. In addition, several algebraic properties of the graph are also determined.

Keywords: orderly set graph, metacyclic $p$-groups, conjugation action

\section{INTRODUCTION}

This paper discusses the relation between graph theory and group theory, usually known as algebraic graph theory. A graph, denoted as $\Gamma$, is a mathematical tool used to connect points and lines. Formally, a graph $\Gamma$ consists of two sets, known as vertices and edges, denoted as $V(\Gamma)$ and $E(\Gamma)$, respectively (Bondy and Murty, 1976).

This paper is constructed with four sections; the Introduction, followed by Methodology, Results and Discussions and ends with Summary of the research.

In this section, a brief introduction regarding graph theory and group theory considered in this research are explained. A graph $\Gamma$ is simple if it has no loops and no two of its links join the same pair of vertices (Bondy and Murty, 1976). In this research, all graphs considered is a simple graph. Next, a graph $\Gamma$ is connected if there exists a path between every pair of distinct vertices (Singh, 2010). In addition, a graph $\Gamma$ is complete if every vertex is connected to every other vertex (Chartrand et al., 2010). The complete graph with $n$ vertices is denoted as $K_{n}$. Meanwhile, the graph $\Gamma$ is called null if it has no vertices, which is denoted by $K_{0}$. If the $n$ number of vertices in $\Gamma$ are not connected, it is denoted by $\bar{K}_{n}$.

In this paper, some metacyclic $p$-groups of odd prime $p$ are considered, in which $p$ is equal to three and five. A metacyclic group is a cyclic group where its factor group is also cyclic. Meanwhile, a metacyclic group with $p$ power order is called a metacyclic $p$-group. There are two presentations of metacyclic $p$-group considered in this paper, namely Type 1 and Type 2, presented in the following theorem. 
Theorem 1. (Basri, 2014) Let $G$ be a non-abelian metacyclic $p$-group. Then $G$ is one of the following:

Type $1 \quad G \cong\left\langle a, b \mid a^{p^{\alpha}}=b^{p^{\beta}}=1,[b, a]=a^{p^{\alpha-\delta}}\right\rangle$, where $p$ is an odd prime number and $\alpha, \beta, \delta \in \square$, $\delta \leq \alpha<2 \delta, \delta \leq \beta, \delta \leq \min \{\alpha-1, \beta\}$.

\section{Type2}

$G \cong\left\langle a, b \mid a^{p^{\alpha}}=1, b^{p^{\beta}}=a^{p^{\alpha-\varepsilon}},[b, a]=a^{p^{\alpha-\delta}}\right\rangle$,

where $p$ is an odd prime number and $\alpha, \beta, \delta, \varepsilon \in \square$, $\delta+\varepsilon \leq \alpha<2 \delta, \delta \leq \beta, \alpha<\beta+\varepsilon$, $\delta \leq \min \{\alpha-1, \beta\}$.

In the next section, the methodology of this research is presented.

\section{LITERATURE REVIEW}

In this section, the methodology of this research is explained. In this research, the set considered is called $\Omega$, which is defined as a set of ordered pairs $(x, y)$ in $G \times G$ such that $\operatorname{lcm}(|x|,|y|)=p, x y=y x, x \neq y$, and $(y, x) \notin \Omega$, where $p$ is considered to be 3 or 5 .

The orderly set graph is extended based on the previous study on the order graph (Payyori and Pasebani, 2014), where the vertex set is the nontrivial subgroups of $G$ and they are joined by an edge if either subgroups divide each other. Meanwhile, the set of vertices of the orderly set graph is the set $\Omega$, where the elements of the set $\Omega$ is of the form $(x, y) \in G \times G$ and the ordered pairs $\left(x_{1}, y_{1}\right)$ and $\left(x_{2}, y_{2}\right)$ are joined by an edge if the ordered pairs in the set $\Omega$ consists of elements with similar order, i.e. $\left|x_{1}\right|=\left|y_{1}\right|$ and $\left|x_{2}\right|=\left|y_{2}\right|$.

Precisely, the definition of the orderly set graph is presented in the following.
Definition 1. Suppose $G$ is a finite non-abelian group and $\Omega$ is a subset of $G \times G$. The orderly set graph, denoted by $\Gamma^{O S}$ is a graph with vertex set $V\left(\Gamma^{O S}\right)=\Omega$, and two vertices $\left(x_{1}, y_{1}\right),\left(x_{2}, y_{2}\right) \in \Omega$ are joined by an edge if and only if $\left|x_{1}\right|=\left|y_{1}\right|$ and $\left|x_{2}\right|=\left|y_{2}\right|$.

In the Results and Discussions section, the orderly set graph will be associated with the metacyclic 3-groups and 5 -groups. Therefore, the following eight lemmas are given which will be used in proving the orderly set graph of the groups, where the elements and the order of the set $\Omega$ in each group are considered.

The first lemma gives the form of elements of order three in metacyclic 3-group of Type 1.

Lemma 1. (Zamri, 2018)Let $G$ be a metacyclic 3-group of Type 1, namely $G \cong\left\langle a, b \mid a^{3^{\alpha}}=b^{3^{\beta}}=1,[b, a]=a^{3^{\alpha-\delta}}\right\rangle, \quad$ where $\alpha, \beta, \delta \in \square, \delta \leq \alpha<2 \delta, \delta \leq \beta, \delta \leq \min \{\alpha-1, \beta\}$ Then, the elements of order three in $G$ are $a^{3^{(\alpha-1) i}}, 1 \leq i \leq 2, b^{3^{(\beta-1)} j}, 1 \leq j \leq 2, a^{3^{(\alpha-1)} i} b^{3^{(\beta-1)} j}$, $1 \leq i \leq 2,1 \leq j \leq 2$, where $\alpha \geq 3$ and $\beta \geq 2$.

The second lemma gives the order of the set $\Omega$ in metacyclic 3-group of Type 1 .

Lemma 2. (Zamri, 2018) Let $G$ be a metacyclic 3-group of Type 1 , namely $G \cong\left\langle a, b \mid a^{3^{\alpha}}=b^{3^{\beta}}=1,[b, a]=a^{3^{\alpha-\delta}}\right\rangle, \quad$ where $\alpha, \beta, \delta \in \square, \delta \leq \alpha<2 \delta, \delta \leq \beta, \delta \leq \min \{\alpha-1, \beta\}$, and

$\Omega=\{(x, y) \in G \times G: \operatorname{lcm}(|x|,|y|)=3, x y=y x, x \neq y\}$ $\backslash\{(x, y)\}$. Then, the order of $\Omega,|\Omega|=36$.

Next, Lemma 3 and Lemma 4 give the elements of order three and the order of the set $\Omega$ in metacyclic $3^{-}$ groups of Type 2 . 
Lemma 3. (Zamri, 2018) Let $G$ be a metacyclic 3-group of Type 2 , namely $G \cong\left\langle a, b \mid a^{3^{\alpha}}=1, b^{3^{\beta}}=a^{3^{\alpha-\varepsilon}},[b, a]=a^{3^{\alpha-\delta}}\right\rangle$, where $\alpha, \beta, \delta, \varepsilon \in \square, \delta+\varepsilon \leq \alpha<2 \delta, \delta \leq \beta$, $\alpha<\beta+\varepsilon, \delta \leq \min \{\alpha-1, \beta\}$. Then, the elements of order three in $G$ is divided into two cases, with the given presentation. For Case (i) where $\alpha=\beta$, the elements are described as follows: $a^{3^{(\alpha-1)} i}, 1 \leq i \leq 2$, $a^{3^{(\alpha-2)} i} b^{3^{(\beta-1)}}, i=2,5,8, a^{3^{(\alpha-2)} i} b^{3^{(\beta-1)} j}$, $i=1,4,7, j=2$, where $\alpha \geq 3, \beta \geq 3$. For Case where $\alpha<\beta$, the elements are described as follows: $a^{3^{(\alpha-1)} i}, 1 \leq i \leq 2, b^{3^{(\beta-1)} j}, 1 \leq j \leq 2$, $a^{3^{(\alpha-1)} i} b^{3^{(\beta-1)} i}, 1 \leq i \leq 2,1 \leq j \leq 2$, where

$\alpha \geq 3, \beta \geq 4$.

Lemma 4. (Zamri, 2018) Let $G$ be a metacyclic 3-group of Type 2, namely $G \cong\left\langle a, b \mid a^{3^{\alpha}}=1, b^{3^{\beta}}=a^{3^{\alpha-\varepsilon}},[b, a]=a^{3^{\alpha-\delta}}\right\rangle$, where $\alpha, \beta, \delta, \varepsilon \in \square, \delta+\varepsilon \leq \alpha<2 \delta, \delta \leq \beta$, $\alpha<\beta+\varepsilon, \delta \leq \min \{\alpha-1, \beta\}$. and $\Omega=\{(x, y) \in G \times G: \operatorname{lcm}(|x|,|y|)=3, x y=y x, x \neq y\}$ $\backslash\{(x, y)\}$. Then, the order of $\Omega,|\Omega|=36$.

The following two lemmas give the elements of order five and the order of the set $\Omega$ in metacyclic 5 -groups of Type 1.

Lemma 5. (Zamri, 2018) Let $G$ be a metacyclic 5-group of Type $1, \quad$ namely $G \cong\left\langle a, b \mid a^{5^{\alpha}}=b^{5^{\beta}}=1,[b, a]=a^{5^{\alpha-\delta}}\right\rangle, \quad$ where $\alpha, \beta, \delta \in \square, \delta \leq \alpha<2 \delta, \delta \leq \beta, \delta \leq \min \{\alpha-1, \beta\}$. Then, the elements of order five in $G$ are described as follows: $a^{5^{(\alpha-1) i}}, 1 \leq i \leq 4, b^{5^{(\beta-1)} j}, 1 \leq j \leq 4$, $a^{5^{(\alpha-1) i}} b^{5^{(\beta-1)} j}, 1 \leq i \leq 4,1 \leq j \leq 4$, where $\quad \alpha \geq 3 \quad$ and $\beta \geq 2$

Lemma 6. (Zamri, 2018) Let $G$ be a metacyclic 5-group of Type 1, namely $G \cong\left\langle a, b \mid a^{5^{\alpha}}=b^{5^{\beta}}=1,[b, a]=a^{5^{\alpha-\delta}}\right\rangle, \quad$ where $\alpha, \beta, \delta \in \square, \delta \leq \alpha<2 \delta, \delta \leq \beta, \delta \leq \min \{\alpha-1, \beta\}$ and

$\Omega=\{(x, y) \in G \times G: \operatorname{lcm}(|x|,|y|)=5, x y=y x, x \neq y\}$ $\backslash\{(x, y)\}$. Then, the order of $\Omega,|\Omega|=300$.

Lastly, Lemma 7 and Lemma 8 present the elements of order five and the order of the set $\Omega$ in metacyclic 5groups of Type 2 .

Lemma 7. (Zamri, 2018) Let $G$ be a metacyclic 5-group of Type 2 , namely $G \cong\left\langle a, b \mid a^{5^{\alpha}}=1, b^{5^{\beta}}=a^{5^{\alpha-\varepsilon}},[b, a]=a^{5^{\alpha-\delta}}\right\rangle$, where $\quad \alpha, \beta, \delta, \varepsilon \in \square, \delta+\varepsilon \leq \alpha<2 \delta, \delta \leq \beta$, $\alpha<\beta+\varepsilon, \delta \leq \min \{\alpha-1, \beta\}$. Then, the elements of order five in $G$ is divided into two cases, with the given presentation. The first case is when $\alpha=\beta$, where the elements are described as follows: $a^{5^{(\alpha-1) i}}, 1 \leq i \leq 4$, $a^{5^{(\alpha-2) j}} b^{5^{(\beta-1)}}, j=4,9,14,19,24, a^{5^{(\alpha-2) k}} b^{5^{(\beta-1)} 2}$, $k=3,8,13,18,23$, $a^{5^{(\alpha-2) m}} b^{5^{(\beta-1)} 3}, m=2,7,12,17,22, a^{5^{(\alpha-2) n}} b^{5^{(\beta-1)} 4}$, $n=1,6,11,16,21$. Next, the second case is when $\alpha<\beta$, where the elements are described as follows: $a^{5^{(\alpha-1)} i}, 1 \leq i \leq 4, b^{5^{(\beta-1)} j}, 1 \leq j \leq 4$, $a^{5^{(\alpha-1) i}} b^{5^{(\beta-1)} j}, 1 \leq i \leq 4,1 \leq j \leq 4$.

Lemma 8. (Zamri, 2018) Let $G$ be a metacyclic 5-group of Type 2, namely $G \cong\left\langle a, b \mid a^{5^{\alpha}}=1, b^{5^{\beta}}=a^{5^{\alpha-\varepsilon}},[b, a]=a^{5^{\alpha-\delta}}\right\rangle$, 
where $\quad \alpha, \beta, \delta, \varepsilon \in \square, \delta+\varepsilon \leq \alpha<2 \delta, \delta \leq \beta$,

$\alpha<\beta+\varepsilon, \delta \leq \min \{\alpha-1, \beta\}$,

and

$\Omega=\{(x, y) \in G \times G: \operatorname{lcm}(|x|,|y|)=5, x y=y x, x \neq y\}$

$\backslash\{(x, y)\}$. Then, the order of $\Omega,|\Omega|=300$.

In the next section, the orderly set graph of metacyclic 3groups and 5 -groups are discussed.

\section{RESULTS AND DISCUSSIONS}

This section presents the main result of this paper, which is the connections between the orderly set graph with some metacyclic $p$-groups of odd prime $p$, where $p$ is equal to 3 and 5 .

Firstly, the orderly set graph, $\Gamma^{O S}$ of metacyclic 3groups are computed. In the following theorems, the orderly set graph of metacyclic 3-groups of Type 1 is given, followed by the metacyclic 3-groups of Type 2 .

Theorem 2.Let $G$ be a metacyclic 3-group of Type 1,

namely $\quad G \cong\left\langle a, b \mid a^{3^{\alpha}}=b^{3^{\beta}}=1,[b, a]=a^{3^{\alpha-\delta}}\right\rangle$,

where

$\alpha, \beta, \delta \in \square, \delta \leq \alpha<2 \delta, \delta \leq \beta, \delta \leq \min \{\alpha-1, \beta\}$, and

$\Omega=\{(x, y) \in G \times G: \operatorname{lcm}(|x|,|y|)=3, x y=y x, x \neq y\}$

$\backslash(\{x, y\})$.If $G$ acts on $\Omega$ by conjugation, the orderly set graph of $G, \Gamma^{O S}=K_{28} \cup \bar{K}_{8}$.

Proof: By Lemma 2, $|\Omega|=36$. Thus, by Definition 1, there are 36 vertices. Next, by Lemma 1, there are eight elements of order three. Based on the set $\Omega$, $(y, x) \notin \Omega$. Since the identity element is order one, there are eight ordered pairs of elements of order one and three in the set $\Omega$. By adjacency of vertices, there are eight isolated vertices. This gives $\bar{K}_{8} \subseteq \Gamma^{O S}$. Next, there are 28 ordered pairs of elements of order three and three in the set $\Omega$. By adjacency of vertices, there are 28 complete components, i.e. $K_{28} \subseteq \Gamma^{O S}$. The proof then follows.

Theorem 3.Let $G$ be a metacyclic 3-group of Type 2, namely

$G \cong\left\langle a, b \mid a^{3^{\alpha}}=1, b^{3^{\beta}}=a^{3^{\alpha-\varepsilon}},[b, a]=a^{3^{\alpha-\delta}}\right\rangle$, where

$\alpha, \beta, \delta, \varepsilon \in \square, \delta+\varepsilon \leq \alpha<2 \delta, \delta \leq \beta$,

$\alpha<\beta+\varepsilon, \delta \leq \min \{\alpha-1, \beta\}$, and

$\Omega=\{(x, y) \in G \times G: \operatorname{lcm}(|x|,|y|)=3, x y=y x, x \neq y\}$ $\backslash(\{x, y\})$. If $G$ acts on $\Omega$ by conjugation, the orderly set graph of $G, \Gamma^{O S}=K_{28} \cup \bar{K}_{8}$.

Proof: By Lemma 4, $|\Omega|=36$. Thus, by Definition 1, there are 36 vertices. Next, by Lemma 3, there are eight elements of order three. Based on the set $\Omega$, $(y, x) \notin \Omega$. Since the identity element is order one, there are eight ordered pairs of elements of order one and three in the set $\Omega$. By adjacency of vertices, there are eight isolated vertices. This gives $\bar{K}_{8} \subseteq \Gamma^{O S}$. Next, there are 28 ordered pairs of elements of order three and three in the set $\Omega$. By adjacency of vertices, there are 28 complete components, i.e. $K_{28} \subseteq \Gamma^{O S}$. The proof then follows.

Next, the orderly set graph of the metacyclic 5-groups of Type 1 and Type 2 are found and presented in the following two theorems.

Theorem 4.Let $G$ be a metacyclic 5-group of Type 1, namely $G \cong\left\langle a, b \mid a^{5^{\alpha}}=b^{5^{\beta}}=1,[b, a]=a^{5^{\alpha-\delta}}\right\rangle$,

where

$\alpha, \beta, \delta \in \square, \delta \leq \alpha<2 \delta, \delta \leq \beta, \delta \leq \min \{\alpha-1, \beta\}$, and 
$\Omega=\{(x, y) \in G \times G: \operatorname{lcm}(|x|,|y|)=5, x y=y x, x \neq y\}$ $\backslash(\{x, y\})$. If $G$ acts on $\Omega$ by conjugation, the orderly set graph of $G, \Gamma^{O S}=K_{276} \cup \bar{K}_{24}$.

Proof: By Lemma 6, $|\Omega|=300$. Thus, by Definition 1, there are 300 vertices. Next, by Lemma 5 , there are 24 elements of order five. By adjacency of vertices, there are 24 isolated vertices, which gives $\bar{K}_{24} \subseteq \Gamma^{O S}$. Next, there are 276 ordered pairs of elements of order one and five in the set $\Omega$. By adjacency of vertices, there are 24 isolated vertices. This gives $\bar{K}_{24} \subseteq \Gamma^{O S}$. Next, there are 276 ordered pairs of elements of order five and five in the set $\Omega$. By adjacency of vertices, there are 276 complete components, i.e. $K_{276} \subseteq \Gamma^{O S}$. The proof then follows.

Theorem 5.Let $G$ be a metacyclic 5-group of Type 2, namely

$G \cong\left\langle a, b \mid a^{5^{\alpha}}=1, b^{5^{\beta}}=a^{5^{\alpha-\varepsilon}},[b, a]=a^{5^{\alpha-\delta}}\right\rangle$, where

$\alpha, \beta, \delta, \varepsilon \in \square, \delta+\varepsilon \leq \alpha<2 \delta, \delta \leq \beta$,

$\alpha<\beta+\varepsilon, \delta \leq \min \{\alpha-1, \beta\}$,

and

$\Omega=\{(x, y) \in G \times G: \operatorname{lcm}(|x|,|y|)=5, x y=y x, x \neq y\}$

$\backslash(\{x, y\})$.If $G$ acts on $\Omega$ by conjugation, the orderly

set graph of $G, \Gamma^{O S}=K_{276} \cup \bar{K}_{24}$.

Proof: By Lemma $8,|\Omega|=300$. Thus, by Definition 1, there are 300 vertices. Next, by Lemma 7, there are 24 elements of order five. Based on the set $\Omega,(y, x) \notin \Omega$. Since the identity element is order one, there are 24 ordered pairs of elements of order one and five in the set $\Omega$. By adjacency of vertices, there are 24 isolated vertices. This gives $\bar{K}_{24} \subseteq \Gamma^{O S}$. Next, there are 276 ordered pairs of elements of order five and five in the set $\Omega$. By adjacency of vertices, there are 276 complete components, i.e. $K_{276} \subseteq \Gamma^{O S}$. The proof then follows.
In addition, several properties of the orderly set graph are also determined which includes the degree of the vertex, the clique number and the chromatic number of the graph, which are presented in the following propositions.

Therefore, the definition of the degree of the vertex, the clique number and the chromatic number of the graph are given in the following.

Definition 2. (Bondy and Murty, 1976) Vertex degrees

The degree of a vertex $v$ in $\Gamma$ is the number of edges of $\Gamma$ incident with $v$, each loop counting as two edges. The minimum and maximum degrees are denoted as $\delta(\Gamma)$ and $\Delta(\Gamma)$, respectively. If the graph is complete, the minimum and maximum degrees of the vertices are the same, usually denoted as $d_{\Gamma}(v)$. Complete graph of $n$ vertices is usually called as $n$-regular graph.

Definition 3. (Balakrishnan, 1997) Clique Number A set $\square$ of vertices in a graph $\Gamma$ is called a clique in $\Gamma$ if there is an edge in $\Gamma$ between every pair of vertices in $\square$ which is itself a complete graph is called a clique. The maximum size of cliques is called the clique number of $\Gamma$, denoted as $\omega(\Gamma)$.

Definition 4. (Chartrand et al., 2010) Chromatic number

The chromatic number of a graph $\Gamma$, usually denoted as $\chi(\Gamma)$, is the smallest number required to colour the vertices where adjacent vertices must be of different colours.

The degree of the vertices of the orderly set graph of metacyclic 3 -groups and 5-groups are given in Proposition 1 and Proposition 2.

Proposition 1. Let $G$ be the metacyclic 3-groups of Type 1 and Type 2 and $\Omega$ is a subset of $G \times G$. If $G$ 
acts $\Omega$ on by conjugation, the degrees of the vertices in $\Gamma$ can be written as follows:

1. The maximum degree of the vertices, $\Delta\left(\Gamma^{O S}\right)=27$.

2. The minimum degree of the vertices, $\delta\left(\Gamma^{O S}\right)=0$.

Proof: From Theorem 2 and 3, the orderly set graph of metacyclic 3 -groups is $K_{28} \cup \bar{K}_{8}$. Since $K_{28}$ is a complete graph, all vertices are adjacent with each other.

By definition of $d_{\Gamma}(v)$, there are 28-1=27 edges incident with each vertex. Thus, the maximum degree of the vertices is 27. Meanwhile, since the graph also consists of eight isolated vertices, i.e. $\bar{K}_{8}$, by definition of $d_{\Gamma}(v)$, the degree of vertices is o. Thus, the minimum degree of the vertices is o. The proof then follows.

Proposition 2. Let $G$ be the metacyclic 5-groups of Type 1 and Type 2 and $\Omega$ is a subset of $G \times G$. If $G$ acts $\Omega$ on by conjugation, the degrees of the vertices in $\Gamma$ can be written as follows:

1. The maximum degree of the vertices, $\Delta\left(\Gamma^{O S}\right)=275$

2. The minimum degree of the vertices, $\delta\left(\Gamma^{O S}\right)=0$

Proof: From Theorem 4and 5, the orderly set graph of metacyclic 3 -groups is $K_{276} \cup \bar{K}_{24}$. Since $K_{276}$ is a complete graph, all vertices are adjacent with each other. By definition of $d_{\Gamma}(v)$, there are 276-1=275 edges incident with each vertex. Thus, the maximum degree of the vertices is 275. Meanwhile, since the graph also consists of 24 isolated vertices, i.e. $\bar{K}_{8}$, by definition of $d_{\Gamma}(v)$, the degree of vertices is o. Thus, the minimum degree of the vertices is o. The proof then follows.
Next, the clique number of the orderly set graph, $\Gamma^{O S}$, denoted by $\omega(\Gamma)$ is given in the following.

Proposition 3. Let $G$ be the metacyclic 3-groups of Type 1 and Type 2 and $\Omega$ is a subset of $G \times G$. If $G$ acts $\Omega$ on by conjugation, the clique number in $\Gamma^{O S}$, $\omega(\Gamma)=28$

Proof: From Theorem 2 and 3, the orderly set graph of metacyclic 3 -groups is $K_{28} \cup \bar{K}_{8}$. Since $K_{28}$ is a complete graph, all vertices are adjacent with each other. Thus, all subgraphs of $K_{28}$ are complete graphs, including itself. By definition of $\omega(\Gamma)$, the largest complete subgraph is $K_{28}$, with maximum size of 28 . Therefore, $\omega(\Gamma)=28$. Meanwhile, since $\bar{K}_{8}$ is a graph with eight isolated vertices, it does not have any complete subgraph. The proof then follows.

Proposition 4. Let $G$ be the metacyclic 5-groups of Type 1 and Type 2 and $\Omega$ is a subset of $G \times G$. If $G$ acts $\Omega$ on by conjugation, the clique number in $\Gamma^{O S}$, $\omega(\Gamma)=276$.

Proof: From Theorem 4 and 5, the orderly set graph of metacyclic 5 -groups is $K_{276} \cup \bar{K}_{24}$. Since $K_{276}$ is a complete graph, all vertices are adjacent with each other. Thus, all subgraphs of $K_{276}$ are complete graphs, including itself. By definition of $\omega(\Gamma)$, the largest complete subgraph is $K_{276}$, with maximum size of 276 . Therefore, $\omega(\Gamma)=276$. Meanwhile, since $\bar{K}_{24}$ is a graph with 24 isolated vertices, it does not have any complete subgraph. The proof then follows.

Lastly, the chromatic number of the orderly set graph 
$\Gamma^{O S}$, denoted by $\chi(\Gamma)$ is presented.

Proposition 5.Let $G$ be the metacyclic 3-groups of Type 1 and Type 2 and $\Omega$ is a subset of $G \times G$. If $G$ acts $\Omega$ on by conjugation, the chromatic number of $\Gamma^{O S}, \chi(\Gamma)=28$.

Proof: From Theorem 2 and 3, the orderly set graph of metacyclic 3 -groups is $K_{28} \cup \bar{K}_{8}$. Since $K_{28}$ is a complete graph, all vertices are adjacent with each other. Thus, by definition of chromatic number, all 28 vertices must be coloured differently so that no adjacent vertices share the same colour. Thus, the chromatic number is 28 . Next, for $\bar{K}_{8}$, since all eight vertices are not adjacent to each other, by definition of chromatic number, any colour from the vertices of $K_{28}$ can be used to colour the vertices. Therefore, the chromatic number, $\chi(\Gamma)=28$. The proof then follows.

Proposition 6. Let $G$ be the metacyclic 5-groups of Type 1 and Type 2 and $\Omega$ is a subset of $G \times G$. If $G$ acts $\Omega$ on by conjugation, the chromatic number of $\Gamma^{O S}, \chi(\Gamma)=276$.

Proof: From Theorem 4 and 5, the orderly set graph of metacyclic 5 -groups is $K_{28} \cup \bar{K}_{8 \text {. Since }} K_{28}$ is a complete graph, all vertices are adjacent with each other. Thus, by definition of chromatic number, all 276 vertices must be coloured differently so that no adjacent vertices share the same colour. Thus, the chromatic number is 276. Next, for $\bar{K}_{24}$, since all 24 vertices are not adjacent to each other, by definition of chromatic number, any colour from the vertices of $K_{276}$ can be used to colour the vertices. Therefore, the chromatic number, $\chi(\Gamma)=276$. The proof then follows.

\section{SUMMARY}

In this paper, the orderly set graph has been introduced, which is denoted by $\Gamma^{O S}$. Later on, the orderly set graph is connected with metacyclic 3-groups and 5-groups in which four main theorems have been found. However, eight lemmas on the elements and the order of the set $\Omega$ in each group have been introduced which later been used in the proving the main theorems. It was found that the orderly set graph of metacyclic $p$-groups where $p$ is an odd prime are found to be a union of complete and empty graph with $n$ number of vertices. In addition, three algebraic properties of the orderly set graph are also determined. The maximum degree of the vertices of $\Gamma^{O S}$ for metacyclic 3-group and 5-group are found to be 27 and 275, respectively. Meanwhile, the minimum degree of the vertices for both groups is 0 . Furthermore, both chromatic and clique number of the metacyclic 3-group is equal to 28. Meanwhile, both chromatic and clique number for metacyclic 5-group is equal to 276 .

\section{ACKNOWLEDGEMENT}

The authors are thankful to Universiti Teknologi Malaysia for the financial support for this research project and Universiti Malaysia Sabah for hosting the Simposium Kebangsaan Sains Matematik (SKSM26). 


\section{REFERENCES}

Balakrishnan, V. (1997). Schaum's Outline of Graph Theory: Including Hundreds of Solved Problems. McGraw Hill Professional.

Basri, A. M. A. (2014). Capability and Homological Functors of Finite Metacyclic $p$-Groups. Universiti Teknologi Malaysia. Ph. D. Thesis.

Bondy, J. A. and Murty, U. S. R. (1976). Graph Theory with Applications. Macmillan London.

Chartrand, G., Lesniak, L. and Zhang, P. (2010). Graphs \& Digraphs. CRC Press.
Payvori, S. and Pasebani, H. (2014). The order graphs of groups. Algebraic Structures and Their Applications. 1(1): 1-10.

Singh, G. S. (2010). Graph Theory. PHI Private Learning Limited. New Delhi.

Zamri, A. N. A. (2018). The Conjugation Degree on a Set of Metacyclic 3 and 5-Groups with Their Related Graphs. Ph.D. Thesis. Universiti Teknologi Malaysia. 